\section{Schwere pulmonale Hypertonie - Vasodilative Therapie in der Lungenstrombahn}

\section{Zusammenfassung}

Die Beeinträchtigung der transpulmonalen Blutströmung bei der chronisch pulmonalen Hypertonie beruht auf einer anhaltenden Verlegung/Verengung des Gefäßquerschnitts. Diese kann einerseits durch eine Vasomotorenreaktion ausgelöst sein, die prinzipiell durch Vasodilatoren antagoniert werden kann, und/oder auf schwer zu beeinflussenden Phänomenen beruhen, wie einer Obliteration des Gefäßlumens (thrombotisches Material) und einem zellulären Umbau der Gefäßwände (vaskuläres Remodelling). Pathophysiologische Konsequenz ist die Überlastung des rechten Ventrikels (chronisches Rechtsherzversagen) sowie eine durch Perfusionsfehlverteilungen bedingte Störung des Gasaustausches. Ziel der neueren Interventionsstrategien ist erstens, die Aufhebung des dauerhaft erhöhten Vasotonus durch Relaxation der glatten Gefäßmuskulatur (Effekt von NO, Kalziumantagonisten, Prostaglandinen); zweitens, eine langfristige Antagonisierung des strukturellen Gefäßumbaus (vaskuläres Remodelling) und drittens, die Verhinderung einer primär oder sekundär eintretenden thrombotisch bedingten Obliteration (Embolie, Insitu-Thrombose). Durch den Einsatz von vasodilatativen Prostaglandinen (Prostazyklin, Iloprost) mit anti-inflammatorisch, anti-proliferativ und anti-aggregativem Wirkspektrum soll eine therapeutische Beeinflussung auch der bislang als irreversibel („fixiert“) erachteten nicht-vasokonstriktiven Widerstandskomponente erreicht werden. Als innovativer Applikationsmodus hat sich in jüngsten Studien die Inhalation zur pulmonalen Anreicherung der Vasodilatanzien (pulmonale Selektivität) mit bevorzugter Deposition gerade in den gut belüfteten Alveolarbezirken (intrapulmonale Selektivität) erwiesen. Die erwünschte Abnahme des pulmonal-vaskulären Widerstandes ist bei inhalativer Applikation, im Gegensatz zur systemischen Anwendung (oral/i.v.), in der Regel nicht mit einer Verschlechterung des Gasaustausches verbunden. Nachteil der Inhalationstherapie mit vasodilatativen Prostanoiden ist die zeitliche Begrenzung des Effekts durch die geringe Halbwertszeit der Substanzen und den raschen Abbau des second-messengers cAMP mittels Phosphodiesterasen. Gegenstand aktueller Forschungsvorhaben ist daher der Versuch, durch simultane Inhibition der Phosphodiesterasen Typ III und IV eine Verlängerung und Verstärkung des Effektes der Prostanoidinhalation zu erzielen.

Pneumologie 2000; 54: 160-169

(c) Georg Thieme Verlag Stuttgart · New York ISSN 0934-8387
F. Grimminger, H. Olschewski, F. Rose, H. A. Ghofrani, N. Weissmann, R. T. Schermuly, D. Walmrath, W. Seeger Medizinische Klinik II, Zentrum für Innere Medizin der Justus-Liebig-Universität Gießen

\section{Pathophysiologie - Vasomotorendysfunktion und vaskuläres Remodelling in der Lunge}

Pathophysiologischer Hintergrund der pulmonalen Hypertonie sind aktive persistierende Vasokonstriktion, Querschnittsverlegung (Obliteration), Gefäßverlust (Rarefikation) und Abnahme der Gefäßelastizität. In der Frühphase der Erkrankung bedingen diese Faktoren zunächst eine Abnahme der funktionellen Gefäßreserven und damit des Gefäßrekruitments unter Belastung, später folgt eine Beeinträchtigung der Lungenperfusion bereits in Ruhe. Ein eingeschränkter aktiver Vasodilatationsmechanismus durch endotheliale Dysfunktion (z.B. mangelhafte Synthese von NO und Prostazyklin) bzw. eine Vasokonstriktion durch inflammatorische Mediatoren (z.B. Endothelin-1, Thromboxan, Serotonin) sind Angriffspunkte der vasodilatativen Therapie (Abb.1). Als pathomorphologisches Korrelat findet sich bei chronisch persistierender pulmonaler Hypertonie eine zunehmende Fixierung der vaskulären Widerstandserhöhung durch quantitative und qualitative Umstrukturierung der Gefäßwände (vaskuläres Remodelling). Weiterhin scheint in den veränderten Lungengefäßen der Mechanismus der Gefäßobliteration durch lokale

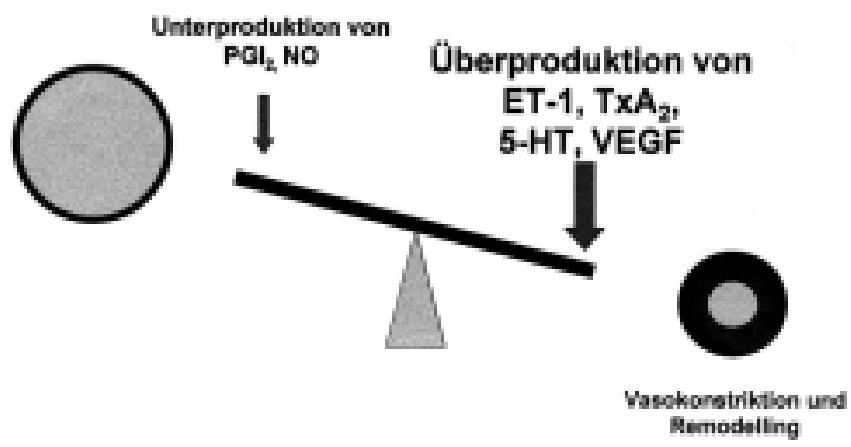

Abb. 1 Störung des Gleichgewichtes vasoaktiver und proliferativer Mediatoren bei der pulmonalen Hypertonie. Das normale Gleichgewicht zwischen den aufgeführten Mediatorengruppen ist bei der pulmonalen Hypertonie zu Gunsten vasokonstriktiver und proproliferativer Faktoren gestört. Die Zunahme von Endothelin-1 (ET-1), Thromboxan $\left(\mathrm{TxA}_{2}\right)$, Serotonin $(5-\mathrm{HT})$ und dem vascular endothelial growth factor (VEGF) bei gleichzeitiger Reduktion von $\mathrm{PGI}_{2}$ und $\mathrm{NO}$ wird als Auslöser des Gefäßwandremodellings angesehen. Darüber hinaus kann die lokale Entstehung weiterer Wachstumsfaktoren und kurzlebiger Metabolite (Tumor Nekrose Faktor, plättchenaktivierender Faktor und Thrombin) beteiligt sein. Die kontinuierliche intravenöse oder inhalative Applikation eines Vasodilatans kann dieses Ungleichgewicht möglicherweise akut antagonisieren und langfristig die Progression des vaskulären Remodellings verlangsamen. 
Thrombozytenaktivierung und In-Situ-Thrombose sekundär verstärkt zu werden. Die grundsätzlichen Mechanismen, die zu diesen pathologischen Gefäßveränderungen führen und deren gemeinsame pathogenetische Endstrecke das vaskuläre Remodelling ist, sind in Abb. 2 dargestellt.
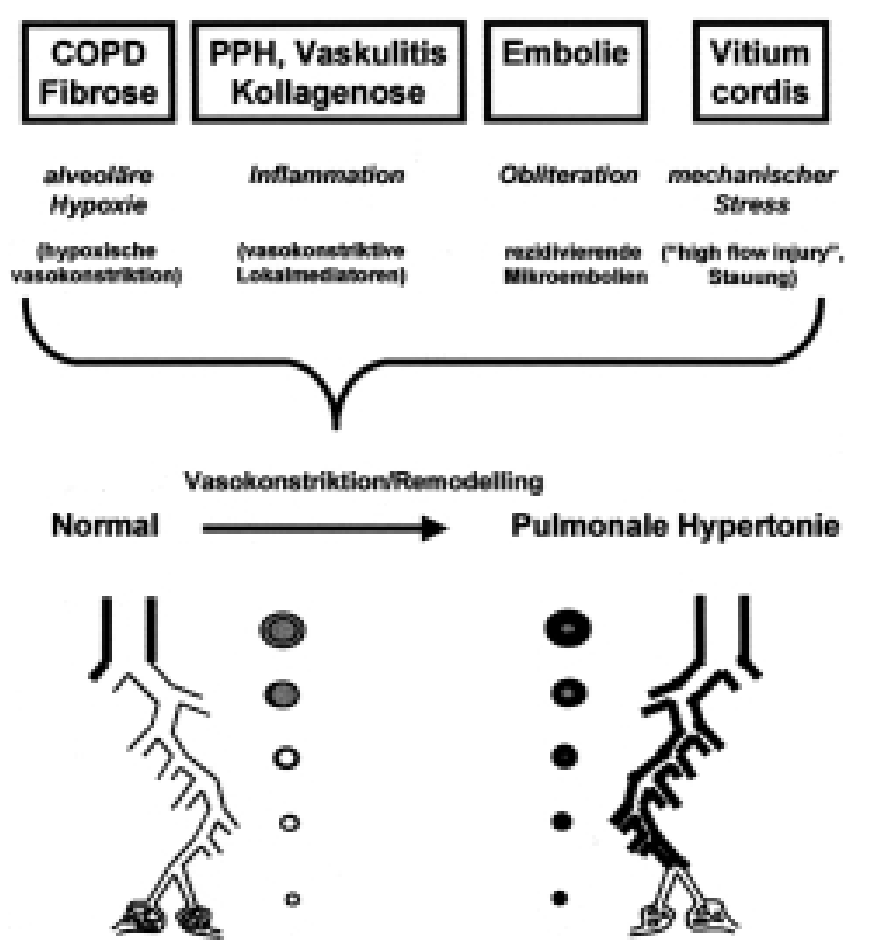

Abb. 2 Grundsätzliche Mechanismen des vaskulären Remodellings: Quantitativ wichtigster Auslöser ist die Daueraktivierung der hypoxischen pulmonalen Vasokonstriktion (HPV). Die chronische alveoläre Hypoxie kann dabei extra-pulmonale Ursachen haben (Bewohner großer Höhen, neurologische Regulationsstörungen, Thoraxdeformitäten), ist jedoch meist auf den Mechanismus der alveolären Hypoventilation bei obstruktiven oder restriktiven Atemwegs- und Lungenerkrankungen zurückzuführen (COPD, Fibrose). Inflammatorische Prozesse können primär extravaskulär oder primär vaskulär lokalisiert sein und spielen eine große Rolle bei Kollagenosen, systemischen Vaskulitiden und der primären pulmonalen Hypertonie (PPH). Die mechanische Obliteration der Gefäße tritt bei akuten und chronisch rezidivierenden Lungenembolien auf (Embolie). Kardiogene Ursachen einer pulmonal vaskulären Druckerhöhung entstehen durch chronische Druck- und Volumenbelastung der Lungenstrombahn z. B. bei Mitralstenose, Vorhofseptumdefekt und chronischer Linksherzinsuffizienz.

\section{Einteilung der pulmonalen Hypertonie}

Die pulmonale Hypertonie ist meistens die Folge einer pulmonalen oder kardialen Erkrankung. Grundsätzliche Mechanismen können sein:

- Daueraktivierung des Mechanismus der hypoxischen Vasokonstriktion durch chronische alveoläre Hypoxie (neurologische Atemregulationsstörung, Thorax- und Skelettdeformitäten, alveoläre Hypoventilation bei obstruktiven oder restriktiven Atemwegs- und Lungenerkrankungen).

- Inflammatorische Lungenerkrankungen mit Gefäßbeteiligung (Kollagenosen, ARDS, Sarkoidose).

- Mechanische Obliteration der Gefäße bei chronisch rezidivierenden oder chronisch persistierenden Lungenembolien.
- Kardiogene Mechanismen einer pulmonal-vaskulären Druckerhöhung mit chronisch venöser Druck- und Volumenbelastung der Lungenstrombahn (Mitralstenose, Vorhofseptumdefekt, chronische Linksherzinsuffizienz).

Sowohl die hypoxischen und entzündlichen als auch die mechanischen Auslösemechanismen sind von strukturellen Veränderungen der Gefäßwände begleitet, die über die akut reversiblen Phänomene der Vasokonstriktion hinaus letztlich eine Abnahme des Gefäßquerschnittes und der Elastizität der Gefäße bedingen (Remodelling). Die aus den dargelegten Mechanismen resultierende Druckerhöhung in der pulmonalen Zirkulation ist gleichbedeutend mit einer erhöhten Nachlast des rechten Herzens. Resultieren hieraus Prozesse der rechtsventrikulären Hypertrophie und/oder der Dilatation, so wird hierfür der Begriff Cor pulmonale benutzt (von der Definition sind kongestive Herzerkrankungen ausgenommen). Ein Cor pulmonale kann sowohl akut als auch chronisch entstanden sein und klinisch kompensiert (ohne Rechtsherzinsuffizienz) oder dekompensiert (mit Rechtsherzinsuffizienz) in Erscheinung treten.

Es lassen sich drei Schweregrade der pulmonalen Hypertonie unterscheiden. Bei der latenten pulmonalen Hypertonie liegt der pulmonal-arterielle Mitteldruck (PAP) bei Ruhe im Normbereich unter $20 \mathrm{mmHg}$, erreicht aber unter Belastung Werte über $28 \mathrm{mmHg}$. Klinisch fällt in der Regel eine Dyspnoe bei stärkerer Belastung und eine mäßige Einschränkung der aeroben Kapazität im Belastungstest auf. Bei der manifesten pulmonalen Hypertonie beträgt der PAP bereits in Ruhe mehr als $20 \mathrm{mmHg}$. Klinisch weisen diese Patienten Dyspnoe bei Belastung und eine erniedrigte aerobe Leistung auf. Die schwere pulmonale Hypertonie ist weniger durch die Höhe des pulmonalen Druckes als vielmehr dadurch charakterisiert, dass schon in Ruhe das Herzminutenvolumen auf Grund der Nachlasterhöhung des rechten Herzens deutlich reduziert ist und unter Belastung kaum ansteigt. Die Patienten sind nur minimal belastbar und bereits in Ruhe findet sich eine venöse Sauerstoffsättigung unter $60 \%$.

Nach der aktuellen WHO-Definition [1] werden die Schweregrade der pulmonalen Hypertonie in Anlehnung an die „New York Heart Association Functional Classification“ eingeteilt:

- Klasse 1 ... Patienten mit pulmonaler Hypertonie ohne Einschränkung der körperlichen Aktivität. Normale körperliche Belastungen führen nicht zu vermehrter Dyspnoe oder Müdigkeit, thorakalen Schmerzen oder Schwächeanfällen.

- Klasse 2 ... Patienten mit pulmonaler Hypertonie mit einer leichten Einschränkung der körperlichen Aktivität. Keine Beschwerden in Ruhe. Normale körperliche Aktivität führt zu vermehrter Dyspnoe oder Müdigkeit, thorakalen Schmerzen oder Schwächeanfällen.

- Klasse 3 ... Patienten mit pulmonaler Hypertonie mit deutlicher Einschränkung der körperlichen Aktivität. Keine Beschwerden in Ruhe. Bereits leichtere als normale Belastungen führen zu Dyspnoe oder Müdigkeit, thorakalen Schmerzen oder Schwächeanfällen.

- Klasse 4 ... Patienten mit pulmonaler Hypertonie mit Unfähigkeit, irgendwelche körperliche Belastung ohne Beschwerden auszuführen. Zeichen der manifesten Rechtsherzinsuffizienz. Dyspnoe und/oder Müdigkeit können be- 
reits in Ruhe vorhanden sein. Bei geringster Aktivität werden die Beschwerden verstärkt.

Klassifikation: Anlässlich der aktuellen PPH-Welt-Konferenz in Evian, 1998, wurde eine neue Nomenklatur und Klassifikation der pulmonalen Hypertonie vorgeschlagen [1].

\section{Pulmonale Arterielle Hypertonie}

1.1 Primäre Pulmonale Hypertonie

a) Sporadisch

b) Familiär

1.2 in Verbindung mit:

a) Bindegewebskrankheiten

b) Links-Rechts-Shuntvitien

c) Portale Hypertonie

d) HIV-Infektion

e) Medikamente/Drogen

1) Appetitzügler

2) andere

f) Persistierende pulmonale Hypertonie der Neugeborenen

\section{Pulmonal Venöse Hypertonie}

2.1 Linksatriale oder linksventrikuläre Erkrankungen

2.2 Linksseitige Klappenerkrankungen

2.3 Kompression der zentralen Lungenvenen

2.4 Mediastinalfibrose

2.5 Adenopathie/Tumoren

2.6 Pulmonale Veno-Okklusive Krankheit

2.7 Andere

3 Pulmonale Hypertonie assoziiert mit Erkrankungen der Atemwege und/oder Hypoxämie

3.1 Chronisch obstruktive Lungenkrankheit

3.2 Interstitielle Lungenkrankheit

3.3 Schlafapnoe

3.4 Erkrankungen mit alveolärer Hypoventilation

3.5 Höhenbewohner

3.6 Lungenkrankheiten der Neugeborenen

3.7 Bronchopulmonale Dysplasie

3.8 Andere

4 Pulmonale Hypertonie auf Grund chronischer thrombotischer und/oder embolischer Erkrankungen

4.1 Thromboembolie der proximalen Lungenarterien

4.2 Obstruktion der distalen Lungenarterien

a) Lungenembolie (Thrombus, Tumor, Parasiten, Fremdkörper)

b) In-situ-Thrombose

c) Sichelzellanämie

5 Pulmonale Hypertonie auf Grund von Erkrankungen, die unmittelbar die Lungengefäße betreffen

5.1 Inflammatorisch

a) Schistosomiasis

b) Sarkoidose

c) Andere

5.2 Pulmonal kapilläre Hämangiomatose

Die Klassifikation fasst in der ersten Katergorie die Primäre Pulmonale Hypertonie mit solchen Krankheitsbildern zusammen, die sich pathomorphologisch und funktionell sehr ähnlich verhalten. Für all diese Krankheitsbilder wird eine genetische Prädisposition postuliert. Auch therapeutisch stel- len die Erkrankungen eine Einheit dar, indem sie alle günstig auf Prostanoide anzusprechen scheinen. Die zweite Kategorie fasst alle Krankheiten zusammen, bei denen die pulmonalvenöse Stauung ursächlich für die pulmonale Hypertonie ist. Diese Erkrankungen sprechen eher ungünstig auf Prostanoide an. Die dritte Kategorie fasst Atemwegs- und Lungenerkrankungen sowie die chronische Hypoxie zusammen. Die gemeinsamen Pathomechanismen sind die hypoxische pulmonale Vasokonstriktion und die Inflammation. Die vierte Kategorie umfasst alle mechanischen Obliterationen der Lungengefäße und bietet sich in erster Linie für chirurgische Interventionen (Pulmonalis-Thrombendarteriektomie) und erst in zweiter Linie für eine medikamentöse Therapie an (abgesehen von einer Antikoagulation). Die fünfte Gruppe fasst die Vaskulitiden und die seltene pulmonal kapilläre Hämangiomatose zusammen.

\section{Diagnostik der chronischen pulmonalen Hypertonie}

Einschwemmkatheter: Gold-Standard in der Diagnostik des chronischen pulmonalen Hypertonus ist der Rechtsherzkatheter. Mit Hilfe des so genannten Swan-Ganz-Ballonkatheters können die wesentlichen Parameter der systemischen und pulmonalen Hämodynamik erfasst werden. Betont werden muss jedoch, dass die reine pulmonal-arterielle Druckmessung zur Erfassung von Frühformen (latente pulmonale Hypertonie) in der Erkrankung nicht ausreicht. Als Kernparameter gilt hier der Lungengefäßwiderstand, welcher bereits bei normalen pulmonal-arteriellen Drucken erheblich von der Norm abweichen kann. Zur Errechnung des Lungengefäßwiderstandes ist die Quantifizierung des Herzminutenvolumens erforderlich. Hierzu dient bei liegendem Rechtsherzkatheter die Farbstoff- oder Thermodilutionsmethode oder das direkte Ficksche Prinzip. Die nichtinvasive Acetylen-Auswasch-Methode scheint ähnlich zuverlässige Ergebnisse $\mathrm{zu}$ liefern.

Liegt eine manifeste pulmonale Hypertonie mit Abweichungen der Ruhewerte vor, sollte eine Reversibilitätsprüfung der Gefäßobstruktion bei liegendem Rechtsherzkatheter erfolgen (Beispiel in Abb. 3). Als Testvasodilatatoren werden Sauerstoff, Calciumantagonisten, Adenosin, Prostazyklin, langlebigere Prostanoide, und Stickstoffmonoxyd verwendet. Als therapeutische Konsequenzen können sich die Langzeitinfusion von Prostazyklin, die chronisch intermittierende Inhalation mit Prostanoiden oder aber die hochdosierte Therapie mit einem Kalziumantagonisten ergeben.

Lungenfunktion: Diese Untersuchung ergibt nur in Ausnahmefällen Hinweise auf das Vorliegen einer chronischen pulmonalen Hypertonie. Zuweilen findet sich eine Einschränkung der pulmonalen Diffusionskapazität. Ansonsten muss bei obstruktiven und restriktiven Lungenerkrankungen sowie Zuständen mit chronischer arterieller Hypoxämie generell mit der Entwicklung eines Cor pulmonale gerechnet werden. Insgesamt lässt sich sagen, dass mit Hilfe der Lungenfunktionsparameter eine Lungengefäßerkrankung weder mit ausreichender Sicherheit diagnostizierbar noch in ihrem Verlauf beurteilbar ist.

EKG: In der Elektrokardiographie nachweisbar sind die chronischen Folgen der pulmonalen Hypertonie in Form eines Cor pulmonale. Allerdings ist eine sichere EKG-Diagnose erst zu 


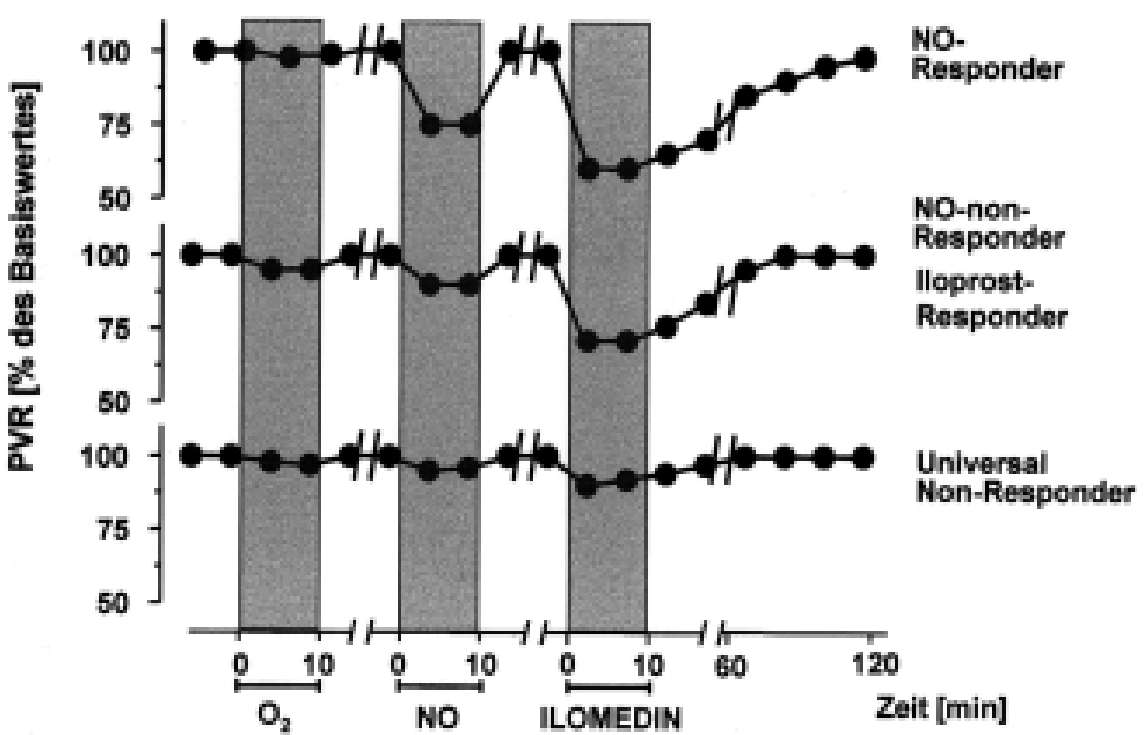

Abb. 3 Reversibilitätsprüfung der pulmonalvaskulären Widerstandserhöhung. Mittels Rechtsherzkatheter wird die pulmonal-vasodilatative Reaktion auf verschiedene Agentien geprüft, hier dargestellt für Sauerstoff $\left(\mathrm{O}_{2}\right)$, NO und aerosoliertes lloprost NOResponder (die immer auch auf aerosoliertes lloprost reagieren), NO-Nonresponder mit vasodilatativer Reaktion auf Iloprost und „Universal“-Non-Responder können unterschieden werden. Der Prozentsatz der auf NO ausgeprägt reagierenden Patienten liegt für PPH bei $<20 \%$, eine ausgeprägte vasodilatative Reaktion auf lloprost findet sich bei dieser Erkrankung jedoch bei $>90 \%$. erwarten, wenn sich die Muskelmasse des rechten Ventrikels etwa verdoppelt hat. Im EKG finden sich dann oftmals Hinweise für eine Rechtsherzhypertrophie bzw. Zeichen der Rechtsherzschädigung. Die klassischen EKG-Kriterien, die jedoch keineswegs obligat sind, umfassen:

- eine Rechtsdrehung der Herzachse (Steil-bis Rechtstyp, ggf. überdrehter Rechtstyp).

- ein RS-Verhältnis in V6 von $<1$

- ein SI QIII Typ

- ein P-pulmonale

- buckelförmig lang deszendierende ST-Strecken-Senkungen in II, III, aVF und V1 - V4.

Bei ca. $20 \%$ der Patienten mit COPD ist mit ventrikulären Rhythmusstörungen zu rechnen, wobei der Schweregrad (Lown-Klassifikation) nicht in Korrelation zu dem Ausmaß der pulmonalen Hypertonie steht.

Thoraxröntgenbild: Der Verdacht auf das Vorliegen einer pulmonalen Hypertonie mit Cor pulmonale kann mit folgenden radiologischen Kriterien untermauert werden:

- Dilatation der Pulmonalarterien und Prominenz des Pulmonalsegmentes.

- Aufweitung des Truncus intermedius der rechten Pulmonalarterie über einen Durchmesser von $18 \mathrm{~mm}$.

- Vergrößerung der sternalen Kontaktfläche in der seitlichen Aufnahme.

- Globale Herzvergrößerung durch rechts und links randbildenden rechten Ventrikel.

Bei gleichzeitigem Lungenemphysem oder schwerer Lungenfibrose sind die radiologischen Zeichen der pulmonalen Hypertonie häufig schwer zu erkennen. Zudem ist zu bedenken, dass viele radiologische Kriterien einer pulmonalen Hypertonie erst in fortgeschrittenem Stadium deutlich werden.

Echokardiographie: Diese Untersuchung ermöglicht die nichtinvasive Diagnose der pulmonalen Hypertonie mit einer sehr hohen Spezifität. Nachweisbar sind:

- Hypertrophie und Dilatation des rechten Ventrikels
- Dilatation des rechten Vorhofes

- paradoxe Septumbeweglichkeit und linksventrikuläre Exzentrizität

- abnorme systolische Zeitintervalle

- abnormes Pulmonalklappenbewegungsmuster sowie Pulmonalinsuffizienz

- Trikuspidalinsuffizienz

- verändertes Strömungsprofil im Ausflusstrakt des rechten Ventrikels.

Die größte Bedeutung kommt der Abschätzung des systolischen pulmonalarteriellen Druckes (PASP) bei vorliegender Trikuspidalinsuffizienz über eine CW-Doppler-Messung des Spitzenwertes des Trikuspidal-Regurgitations-Jets zu, nach der Gleichung: PASP $=4(\mathrm{~V})^{2}+5 \mathrm{mmHg}$ (V= peak velocity (in $\mathrm{m} / \mathrm{s}$ ) der Trikuspidalklappen-Regurgitation; $5 \mathrm{mmHg}=$ angenommener Druck im rechten Vorhof. Eine Weiterentwicklung stellt die Anwendung dieser Methode bei ergometrischer Belastung zur Aufdeckung einer latenten pulmonalen Hypertonie dar.

Die transösophageale Echokardiographie ist für die Darstellung des Vorhofseptums (Vorhofseptumdefekt/offenes Foramen ovale/fehleinmündende Lungenvene) sowie intrakavitärer Thromben von Bedeutung.

Laborchemische Diagnostik: Bei Rechtsherzdekompensation kann eine massive Erhöhung der Leberwerte auftreten, bei kardiogenem Schock auf Grund des Rechtsherzversagens finden sich Laktaterhöhung und Azidose. Ansonsten existieren keine spezifischen Laborparameter der pulmonalen Hypertonie. Extrahierbare Kernantigene sind hilfreich zum Nachweis einer spezifischen Kollagenose.

Ventilations-Perfusionsszintigraphie/Pulmonalisangiographie/Spiral-CT: Diese radiologischen Untersuchungsverfahren dienen dem Ausschluss bzw. der Diagnosesicherung und evtl. Operationsvorbereitung bei Cor pulmonale infolge einer chronisch rezidivierenden oder einer chronisch persistierenden Embolie. Spiral-CT-Untersuchungen mit Kontrastmittel stellen eine bildgebende Alternative zur Pulmonalisangiographie 
dar und sind dieser bei Verdacht auf Embolien der zentralen Gefäße überlegen. Die Entwicklung der Aussagefähigkeit der Kernspintomographie für diese Fragestellungen bleibt abzuwarten.

Screening-Untersuchung: Bestimmte Risikogruppen sollten vorsorglich untersucht werden, auch wenn keine Krankheitszeichen vorliegen. Solche mit einer familiären Belastung, HIVInfektion, Leberzirrhose (zumindest vor geplanter Lebertransplantation) und Bindegewebskrankheiten aus dem Formenkreis der Sklerodermie. Weiterhin sollte bei Patienten mit Gerinnungsanomalien (Lupus antikoagulans, Protein-S-/Protein-C-Mangel, APC-Resistenz) oder stattgehabten Lungenembolien das Vorliegen einer chronisch pulmonalen Hypertonie ausgeschlossen werden. Als Risikopatienten gelten auch Patienten mit fortgeschrittener COPD und Patienten mit nicht korrigierten Mitralklappenvitien oder Rezirkulationsherz (Vorhofseptumdefekt/Ventrikelseptumdefekt).

Das Screening sollte Anamnese, körperliche Untersuchung, Lungenaufnahme, EKG, Lungenfunktion und Echokardiographie umfassen. Eine Spiroergometrie ist bei typischer Anamnese und negativer Primärdiagnostik indiziert. Bei begründetem Verdacht auf das Vorliegen einer pulmonalen Hypertonie werden weitere Maßnahmen wie Ventilations-PerfusionsSzintigraphie, Pulmonalis-Angiographie und Einschwemmkatheteruntersuchung (ggf. unter ergometrischer Belastung) notwendig.

\section{Therapie der chronischen pulmonalen Hypertonie des Cor pulmonale}

Im Vordergrund der Therapie steht neben der Vermeidung der auslösenden Faktoren bzw. der pulmonalen oder kardialen Grunderkrankung die Senkung des pulmonal-arteriellen Druckes und damit die Reduktion der rechtsventrikulären Nachlast. Die potenziell reversiblen Komponenten der Gefäßquerschnittsverminderung sind Angriffspunkte der vasotropen Therapie.

\section{Vasotrope Therapie}

Prinzipiell bestehen zwei Möglichkeiten, durch pharmakologische Intervention den Gefäßquerschnitt zu erweitern. Aufhebung eines dauerhaft erhöhten Vasotonus durch Relaxation der glatten Gefäßmuskulatur: (akuter Effekt von Vasodilatatoren), Beeinflussung des strukturellen Gefäßumbaus (vaskuläres Remodelling) durch Einsatz von antiinflammatorischen und antiproliferativen Agenzien. Die Annahme erscheint naheliegend, dass diejenigen Patienten auf Vasodilatatoren am besten ansprechen, bei denen eine akut reversible Komponente der vaskulären Widerstandserhöhung im Sinne einer Vasokonstriktion im Rahmen der Reversibilitätstestung identifiziert werden kann. Diese Annahme wird jedoch durch Beobachtungen eines Teilkollektivs von Patienten mit primärer pulmonaler Hypertonie infrage gestellt, bei denen trotz geringer akuter Ansprache auf vasodilatative Prostanoide eine mittelfristige Verbesserung unter chronischer Applikation dieser Agentien beobachtet werden konnte. Selbst bei primär mechanischer Obliteration (z.B. chronische Lungenembolien) oder ausgeprägter Gefäßrarifizierung (z.B. schweres Lungenemphysem) kann durch sekundäre Remodelling-Prozesse in den verbliebenen (hyperperfundierten) Gefäßarealen eine
Aggravierung des Krankheitsbildes auftreten, die einer vasotropen Therapie zugänglich ist. Momentan werden folgende Substanzen zur Beeinflussung des pulmonalen Vasotonus angewendet bzw. befinden sich in der klinischen Erprobung:

- Kalziumantagonisten

- inhalatives Stickstoffmonoxyd

- Prostazyklin intravenös

- Iloprost intravenös/inhalativ

- Sauerstofflangzeittherapie

Vermutet wird, dass die Prostanoide, zusätzlich zu dem akut muskelrelaxierenden Effekt, auch über einen verzögert einsetzenden Anti-Remodelling-Effekt verfügen. Diese Komponente der Gefäßquerschnittserweiterung dürfte allerdings erst nach einer zellulären Umstrukturierung in der Gefäßwand messbar werden und somit ein längeres Behandlungsintervall erfordern.

\section{Kalziumantagonisten}

Die beiden Kalziumantagonisten, die bislang am häufigsten Verwendung finden in der Behandlung der chronischen pulmonalen Hypertonie, sind Nifedipin und Diltiazem $[3,4]$. Bei primärer pulmonaler Hypertonie besteht keine generelle Indikation für Kalziumantagonisten. Bei einer Untergruppe von Patienten allerdings, die bei der akuten Testung mit einer ausgeprägten Senkung des pulmonalen Drucks und Widerstandes reagierten, führten hochdosierte Kalziumantagonisten $\mathrm{zu}$ einer eindrucksvollen klinischen, hämodynamischen und prognostischen Besserung [4]. Zur Umgehung der sehr zeitaufwendigen und wegen möglichen systemischen hämodynamischen Effekten nicht ungefährlichen Testung der pulmonalen Vasoreaktivität auf Kalziumantagonisten hat sich klinisch die schnell und risikoarm durchführbare Testung der Gefäßreagibilität mit inhalativem Stickstoffmonoxyd (NO) bewährt [2]. In vielen Zentren wird gegenwärtig auf der Basis dieses Testes (Abfall des pulmonalarteriellen Widerstandes unter NO um $>30 \%$ auf ein Minimum unterhalb 800 dyn $\cdot \mathrm{s}$. $\mathrm{cm}^{-5}$ die Indikation für einen Therapieversuch mit einem hochdosierten Kalziumantagonisten gestellt [2,3]. Der Prozentsatz der so definierten NO/Kalziumantagonisten-Responder liegt allerdings bei $<20 \%$ aller Patienten mit PPH. Nach oraler Gabe von $20 \mathrm{mg}$ Nifedipin bzw. $60 \mathrm{mg}$ Diltiazem wird die Dosierung langsam gesteigert bis zum Erreichen des Hochdosisbereiches oder bis zum Auftreten von Nebenwirkungen. Es zeigte sich, dass sehr hohe Dosierungen von Kalziumkanalblockern notwendig sind, um eine langfristige klinische Stabilisierung zu erreichen [3]. In einigen Fällen ist eine Dosierung von Nifedipin um $250 \mathrm{mg}$ und von Diltiazem um $700 \mathrm{mg}$ erforderlich. Wesentliche Nachteile der oralen Kalziumantagonisten sind einerseits ihre möglichen Effekte auf die systemische Zirkulation im Sinne einer peripheren Vasorelaxation mit Hypotension und andererseits ein Abfall des Herzzeitvolumens auf Grund der negativ inotropen Effekte (insbesondere bei Diltiazem). Weiterhin kann sich ein nachhaltiger Effekt auf den Gasaustausch in Form einer Zunahme der Ventilations-Perfusionsverteilungsstörungen durch die unselektive Vasodilatation in der Lungenstrombahn manifestieren. Wenige klinische Erfahrungen liegen bislang mit dem neuen Kalziumkanalblocker Felodipin vor, welcher ein potenter Vasodilatator ist, gleichzeitig aber weit weniger negativ inotrope Effekte verursacht. 


\section{Therapiestudien mit inhalativem NO}

Unter der Vorstellung, dass bei Patienten mit pulmonaler Hypertonie eine Störung der endothelialen NO-Synthese vorliegt $[5,6]$, wurde der therapeutische Effekt einer exogenen NO-Substitution untersucht. NO hat keinen wesentlichen zusätzlichen vasodilatativen Effekt in der normalen pulmonalen Zirkulation, kann aber den pulmonal-vaskulären Widerstand bei Patienten mit chronischer pulmonaler Hypertonie senken, wenn eine Teilkomponente der Gefäßobstruktion auf einer reversiblen Tonuszunahme der glatten Gefäßmuskulatur beruht. Für die klinische Anwendung der pulmonalen Hypertonie von Stickstoffmonoxyd werden bislang folgende Indikationen überprüft:

- Kurzzeitanwendung zur hämodynamischen Rekompensation bei akuter pulmonaler Hypertonie mit akuten schweren Störungen der Ventilations-Perfusions-Verteilung (ARDS, akute Lungenembolie, Pneumonie)

- Kurzzeitanwendungen zur Überwindung einer akuten pulmonal-hypertensiven Krise bei vorbestehender chronischer pulmonaler Hypertonie

- persistierende pulmonale Hypertonie des Neugeborenen

- Schnelltest der pulmonalen Gefäßreagibilität (siehe oben)

- Überbrückung der terminalen Phase bei Patienten mit chronischer pulmonaler Hypertonie in der Transplantationsvorbereitung

- Langzeitinsufflation zur Dauerbehandlung der chronischen pulmonalen Hypertonie (Perspektive umstritten).

Problematisch ist die Sicherstellung einer kontinuierlichen NO-Zufuhr beim nichtbeatmeten Patienten. Hierbei ist insbesondere von Bedeutung, dass bei akuter Unterbrechung einer kontinuierlichen NO-Zufuhr in kürzester Zeit eine (potentiell lebensbedrohliche) pulmonal-hypertensive Krise auftreten kann (Rebound-Phänomen). Zudem ist NO ein extrem labiles und reaktives Gas mit potenzieller Toxizität. Es gibt Hinweise, dass im alveolären Kompartiment eine Beeinträchtigung der Surfactantfunktion, des epithelialen Ionentransportes sowie lokal immunkompromittierende Effekte auftreten können [7-9]. Gegenwärtig werden technische Entwicklungen überprüft, die geringe Mengen von inhalativem NO als „Bolus“ in einem kurzen Zeitfenster der Inspiration applizieren, um die Dosis bei hoher Effektivität gering zu halten. Zudem wird die Möglichkeit einer alveolären Deposition von aerosolierten NO-Liberatoren zur lokalen NO-Langzeitabgabe in experimentellen Modellen untersucht.

\section{Prostazyklin intravenös}

$\mathrm{PGI}_{2}$ ist ein Metabolit der Arachidonsäure mit ausgeprägter vasodilatativer Wirksamkeit auch in der Lungenstrombahn. Es hat eine kurze Halbwertszeit, wird aber im Gegensatz zu NO im Plasma erst nach zwei bis drei Minuten inaktiviert, so dass mehrere Passagen in der Lungenstrombahn möglich sind. Vor dem Hintergrund, dass im Lungengewebe von Patienten mit primärer und sekundärer pulmonaler Hypertonie wiederholt eine deutliche Verminderung der endothelialen Prostazyklinsynthese beobachtet werden konnte, wurde der Mangel an Prostazyklin zu einem pathogenetischen Faktor der chronischen pulmonalen Hypertonie deklariert [10]. In zwei kontrollierten Studien und einer Vielzahl von Anwendungsbeobachtungen wurde gezeigt, dass intravenöses Prostazyklin die Hämodynamik, Belastbarkeit und Mortalität von Patienten mit PPH verbessert [11 - 13]. Das Gleiche gilt offenbar für das chemische Analogon Iloprost, allerdings ist die Datenbasis hierfür schmaler. Prostazyklin ist in den USA und einigen europäischen Ländern zur Therapie der schweren pulmonalen Hypertonie im NYHA Stadium III-IV zugelassen. Das Medikament wird über eine tragbare Infusionspumpe entweder über einen implantierten Port oder Hickman-Katheter infundiert. Über die vasodilatativen Effekte hinaus könnte der therapeutische Wert des Prostazyklins in einer Modulation des vaskulären Proliferationsverhaltens sowie der Prävention einer pulmonalvaskulären Thrombosierung liegen und somit den Prozess des vaskulären Remodellings günstig beeinflussen. Die Überlegenheit der Prostazyklins gegenüber Kalziumantagonisten beruht zudem wahrscheinlich auf der Abwesenheit von negativ inotropen Effekten. Die Dosierung des Prostazyklins wird anhand der typischen Nebenwirkungen (Kieferschmerzen/Kopfschmerzen/Durchfälle) festgelegt.

Als wesentlicher Nachteil der intravenösen Prostazyklintherapie muss angesichts der hohen Kosten des Präparates die Notwendigkeit einer Dosissteigerung zur Wirkungserhaltung in der Langzeittherapie angesehen werden. Eine sich über Jahre entwickelnde Tachyphylaxie macht in Extremfällen eine Erhöhung der Infusionsdosis von initial $2-5 \mathrm{ng} / \mathrm{kg} / \mathrm{min}$ auf weit über $100 \mathrm{ng} / \mathrm{kg} / \mathrm{min}$ erforderlich, was mit extrem hohen Therapiekosten verbunden ist. Nach 1 Jahr Behandlungsdauer liegt die durchschnittliche Infusionsmenge in den US-amerikanischen Zentren bei $20-40 \mathrm{ng} / \mathrm{kg} / \mathrm{min}$ [2]. Zudem ist die systemische (intravenöse) Applikation eines vasodilatativen Prostanoids mit folgenden Nachteilen verbunden:

- Antagonisierung der hypoxischen pulmonalen Vasokonstriktion und damit Zunahme der Ventilations-PerfusionsVerteilungsstörung und konsekutivem Abfall der arteriellen Oxygenierung

- unerwünschte systemische Effekte der Vasodilatation (Hypotension, Kieferschmerzen, Kopfschmerzen, Durchfälle)

- die Möglichkeit von Katheterinfektionen mit der Gefahr der Sepsis und der Katheterthrombosierung

- die Gefahr der pulmonal-hypertensiven Krise mit akuter Rechtsherzdekompensation bei Unterbrechung der Dauerinfusion.

Zusammenfassend hat die intravenöse Prostazyklintherapie ihren gesicherten Stellenwert bislang nur in der Behandlung der primären pulmonalen Hypertonie. Die beschriebenen Nachteile machen die Entwicklung alternativer Applikationsformen der Prostanoide wünschenswert.

\section{Inhalative Applikation von Prostanoiden}

Ziel dieses Ansatzes ist es, die Notwendigkeit einer kontinuierlichen Infusion zu vermeiden, und eine selektive pulmonale Vasodilatation zu erreichen. Im Gegensatz zum intravenösen Prostazyklin ist bei inhalativer Applikation von Iloprost unter Ausnutzung der räumlichen Nachbarschaft der Alveolen zu den pulmonalen präkapillären Widerstandsgefäßen (Abb.4) eine preferentielle pulmonale Vasodilatation in den gut ventilierten (aerosolzugänglichen) Arealen zu erwarten. Mit der Aerosol-Applikation von Prostazyklin wie auch Iloprost (lang wirkendes Prostazyklin-Analogon) wird bei Patienten mit schwerer pulmonaler Hypertonie eine der intravenösen Applikation von Prostazyklin vergleichbare pulmonale Vasodilatation erreicht (Absenkung des pulmonalvas- 


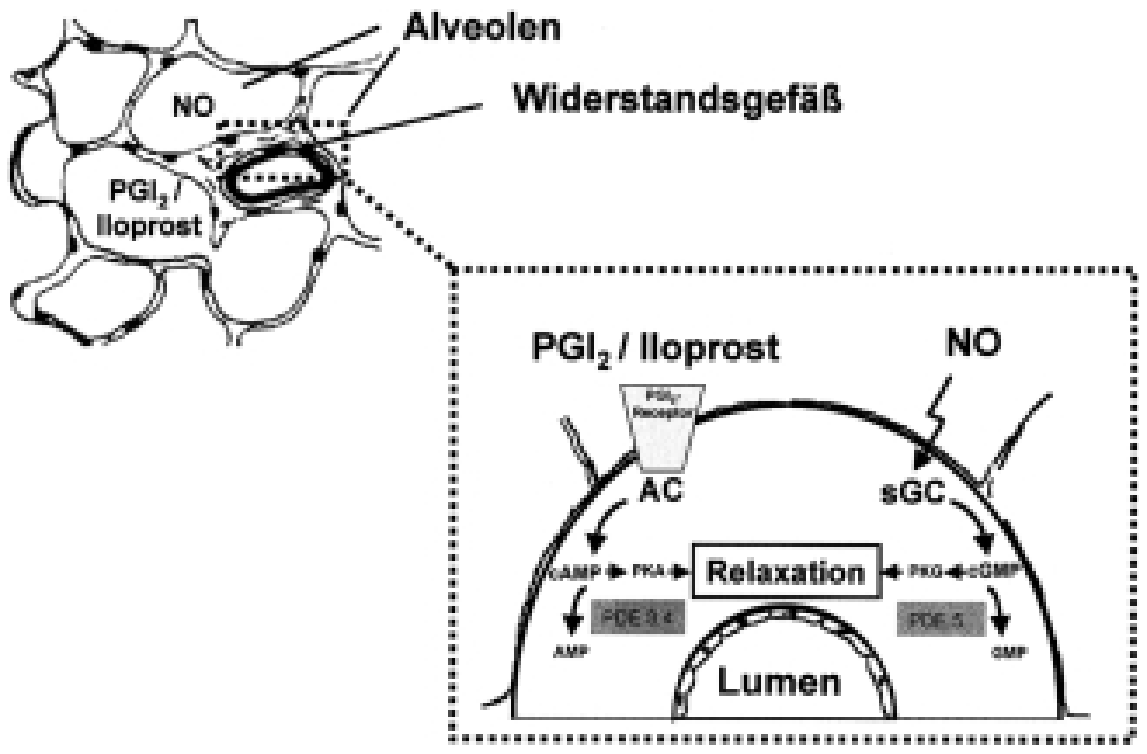

Abb. 4 Wirkmechanismus der inhalativen Vasodilatatoren an den präkapillären Widerstandsgefäßen der Lunge. Der inhalative Zugang ermöglicht die selektive Anreicherung der eingesetzten Vasodilatatoren in den gut ventilierten Alveolarbezirken. Vermittelt wird der vasodilative Effekt in den Muskelzellen präkapillärer Arteriolen über die second-messenger zyklisches Adenosinmonophosphat (CAMP), nach Stimulation der Adanylatzyklase (AC) durch Prostazyklin/ lloprost, und Guanosinmonophosphat (cGMP), nach Stimulation der löslichen Guanylatzyklase (sGC) durch NO. Die zyklischen Nukleotide führen über eine Aktivierung der Proteinkinasen (PK-A und PK-G) und über die Herabsetzung der zytoplasmatischen Kalziumspiegel zu einer Hemmung der AktinMyosin-Interaktion und damit zu einer Abnahme des Vasotonus $[7,17]$. Der relaxierende Effekt wird durch den raschen Abbau der second-messenger durch jeweils spezifische Phosphodiesterasen (PDE 3,4,5) begrenzt.

kulären Widerstandes zwischen $20 \%$ und $>50$ [14]), wohingegen die nachteiligen Effekte des intravenösen Prostazyklins auf den systemischen Blutdruck und den Gasaustausch weitgehend vermieden werden. Allerdings klingt auch nach Verwendung des länger wirkenden Iloprost der pulmonal vasodilatative Effekt nach $1-2 \mathrm{~h}$ ab, was zur Aufrechterhaltung der Wirkung vielfache Aerosolierungen verlangt. Langzeitbeobachtungen mit täglicher Aerosol-Applikation von Iloprost (6-9 Inhalationen über den Tag verteilt; tägliche Iloprost-Gesamtdosis zwischen 50 und $150 \mu g$; Abb. 5) liegen an über 200 Patienten vor, mit der längsten Beobachtungszeit von über 5 Jahren. Bei Umrechnung auf das Körpergewicht und Angabe pro Zeit ergibt sich hieraus, dass der pulmonalvasodilatative Effekt mit einer Dosierung von nur $1-2 \mathrm{ng} / \mathrm{kg} /$ min Iloprost erzielt wird. Interessant ist zudem, dass bei diesem Applikationsmodus kaum eine Tachyphylaxie beobachtet wird. Da die Wirkung des inhalativ applizierten Iloprost nur langsam abklingt, treten nach bisheriger Beobachtung keine wesentlichen Rebound-Phänomene auf. Gegenwärtig wird der Effekt dieser Therapie auf Belastbarkeit und Mortalität bei Patienten mit primärer und sekundärer pulmonaler Hypertonie in einer randomisierten, doppelblinden multizentrischen Studie überprüft. Zum jetzigen Zeitpunkt sollte dieses inhalative Therapieverfahren bevorzugt im Rahmen klinischer Studien oder in begründeten Heilversuchen in den hierfür ausgewiesenen Zentren zur Anwendung kommen. Ein Algorithmus, wie er in dem hiesigen Zentrum Verwendung findet, ist in Abb. $\mathbf{6}$ wiedergegeben.

Es muss betont werden, dass bei kapillärer und postkapillärer Gefäßokklusion bei Anwendung jeglicher vasodilativer Therapie die Gefahr eines akuten Lungenödems auf Grund eines relativen Überwiegens der Vasodilatation an den präkapillären Widerstandsgefäßen bei gleichzeitigem Fortbestehen des fixierten hohen postkapillären Gefäßwiderstands mit konsekutiver Erhöhung des kapillären Filtrationsdruckes besteht.

Zusammenfassend stellt die inhalative Iloprost-Applikation somit eine sehr interessante therapeutische Maßnahme zur preferentiellen pulmonalen Vasodilatation dar, mit einem

\section{Inhalationsschems}

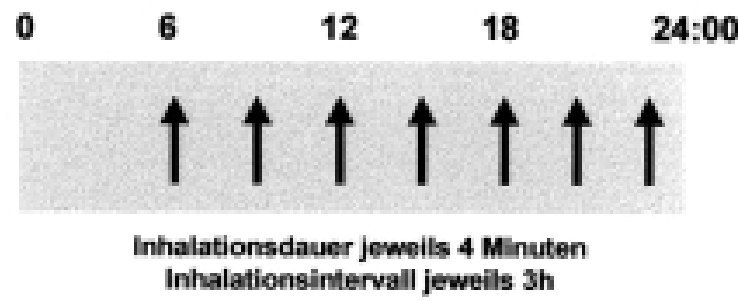

Aufbau eines Inhalationsystems (Uitraschallvernebler)

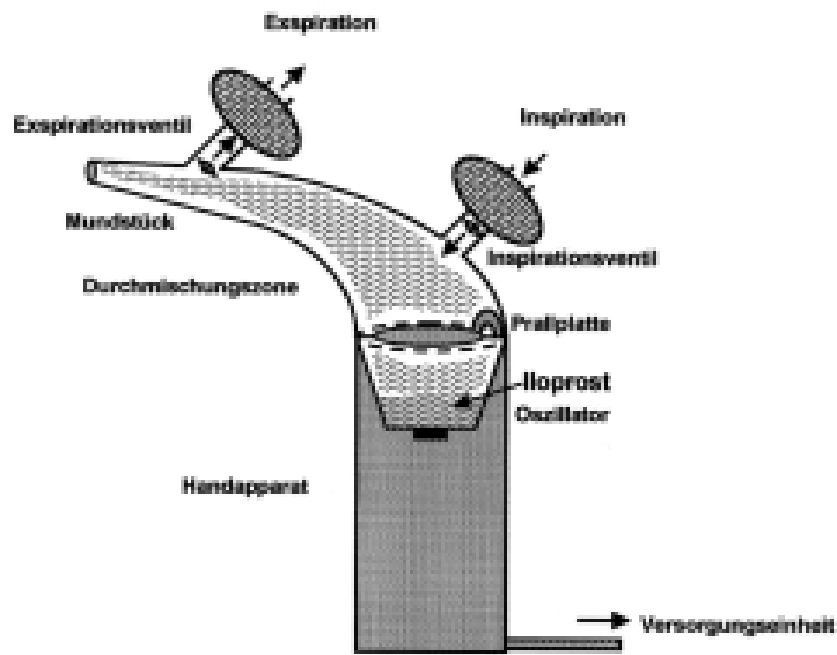

Abb. 5 Beispiel eines Protokolls und eines Verneblersystems be inhalativer Applikation von Iloprost. Nach dem vorliegenden Schema erfolgen jeweils 6 inhalative lloprost-Applikationen über den Tag verteilt. Bei der dargestellten Ultraschalltechnik (Multisonic Ultraschallvernebler von Schill) wird das lloprost in einer Konzentration von $5 \mu \mathrm{g} / \mathrm{ml}$ im Vernebler angeboten. Bei 4-minütiger Inhalation, einer Aerosolgenerierung von $163 \mu \mathrm{l} / \mathrm{min}$ und einer Effektivität der aerosolierten Flüssigkeit (gemessen am Mundstück) von ca. $86 \%$ ergibt sich als inhalierte Einzeldosis eine lloprost-Menge von ca. 2,8 $\mu \mathrm{g}$. Durch Filtersysteme wird eine Kontamination der Umgebung weitgehend vermieden. 
Basistherapie: Antikoagulation, Diuretika bei Ödemen, Sauerstoff bei Hypoxämie

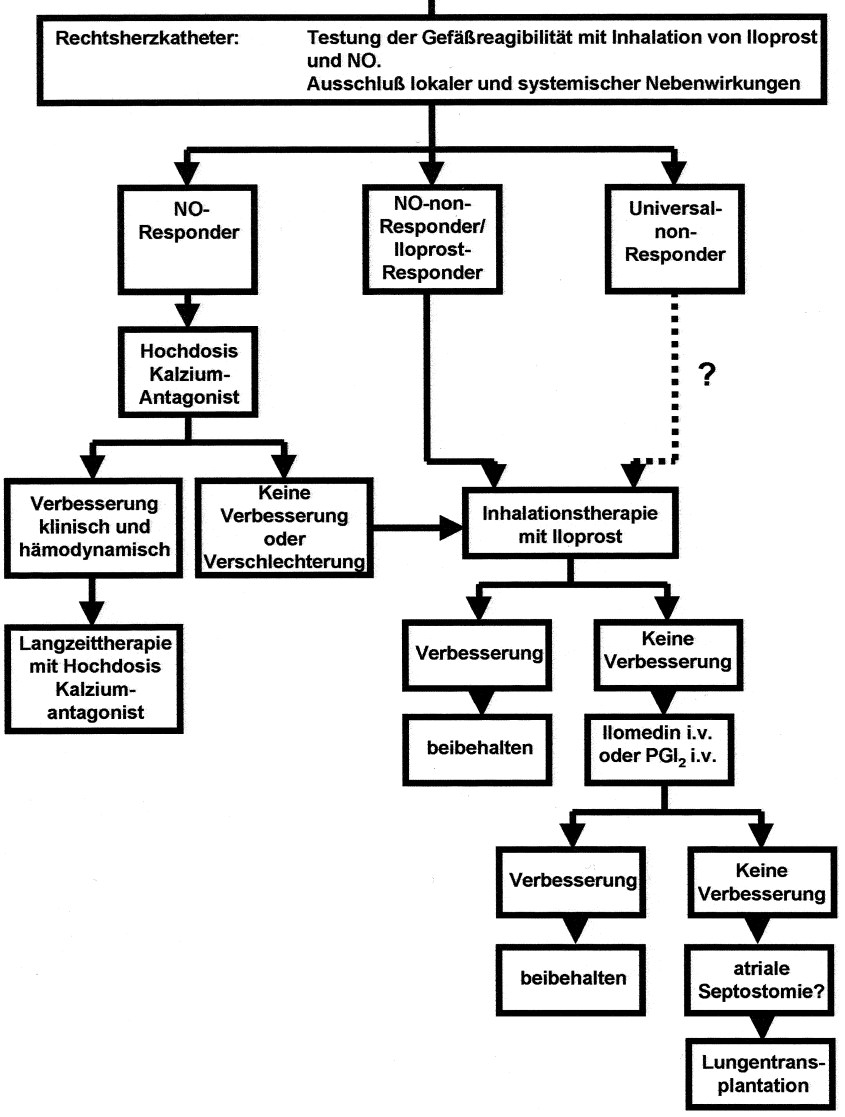

Abb. 6 Algorithmus bei primärer pulmonaler Hypertonie. Das Vorgehen der Autoren bei begründeten Heilversuchen ist dargestellt.

verminderten Risiko von systemischen Nebenwirkungen. Auf Grund der günstigen Beeinflussung der Ventilations-Perfusions-Verteilung in der Lunge profitieren könnten insbesondere auch Patienten mit einer präexistenten Gasaustauschstörung, z.B. sekundäre pulmonale Hypertonien bei Fibrosen [15], profitieren. Neben der Langzeitanwendung in stabilen Krankheitsphasen der schweren pulmonalen Hypertonie stellt die Inhalation von Iloprost nach bisher vorliegenden Daten auch ein Instrument zur Überwindung einer pulmonal-hypertensiven Krise dar. Nach den bisherigen Erfahrungen muss auch erwogen werden, selbst Non-Responder (fehlende akute pulmonale Vasodilatation auf Iloprost-Inhalation) einer Langzeittherapie zuzuführen, da es unter dieser Therapie bei einem Teil der Patienten zu einer klinisch relevanten Wiederherstellung der Gefäßreagibilität im Sinne einer Fähigkeit zur Vasodilatation, möglicherweise mit Regredienz des vaskulären Remodelling kommt [12]. Vermutet wird, dass hierbei insbesondere proliferationshemmende Eigenschaften der Prostanoide eine Rolle spielen. Darüber hinaus hemmt Prostazyklin die Thrombozytenaggregation und weitere inflammatorisch kompetente Zellen und reduziert somit möglicherweise Entzündungsmechanismen, die der Gefäßreaktion bei chronisch persistierender pulmonaler Hypertonie zu Grunde liegen. So konnte in Experimenten mit neutrophilen Granulozyten von Patienten mit primärer und sekundärer pulmonaler Hypertonie nach der Inhalation mit Iloprost eine anhaltende Herunterregulierung der inflammatorischen granulozytären
Aktivität beobachtet werden. Folglich stellt dieses Konzept zusätzlich einen Therapieansatz zur Reduktion der perpetuierenden Entzündungsmechanismen dar, die der Gefäßreaktion bei der pulmonalen Hypertonie zugrunde liegen.

\section{Sauerstofflangzeittherapie}

Die Sauerstofflangzeittherapie der chronischen pulmonalen Hypertonie ist bei medikamentös nicht $\mathrm{zu}$ bessernder arterieller Hypoxie (unzureichender Effekt von Antiobstruktiva, Antibiotika) mit einem arteriellen Sauerstoffpartialdruck von weniger als $65 \mathrm{mmHg}$ in Ruhe angezeigt. Zu den Effekten des Sauerstoffs gehört die Senkung des pulmonalvaskulären Widerstandes bei den Formen der sekundären pulmonalen Hypertonie, für die eine alveoläre Hypoxie einen wesentlichen Trigger der pulmonalvaskulären Widerstandserhöhung darstellt. Die größte Untergruppe stellen hierbei die Patienten mit chronisch obstruktiven Lungenerkrankungen (COPD) dar. In der $\mathrm{O}_{2}$-Langzeittherapie dieser Patienten lässt sich eine signifikante Besserung des Hämatokrits und der rechtsventrikulären Pumpfunktion nachweisen. Lebensqualität und psychomotorische Funktionen verbessern sich, während Krankenhaustage und Arztbesuche abnehmen. Um effektiv zu sein, muss die Sauerstofflangzeittherapie jedoch für mindestens 16 Stunden/Tag durchgeführt werden. Wichtig ist insbesondere die Applikation während der Nacht, wenn die arterielle Hypoxämie und damit die hypoxische Vasokonstriktion durch Hypoventilation erheblich aggraviert sein kann. Bei einer Überlagerung durch Atemregulationsstörungen muss die Notwendigkeit einer dauerhaften nächtlichen CPAP-Beatmung überprüft werden. Die Sauerstoffapplikation erfolgt über einoder zweilumige Nasensonden. Bei Notwendigkeit einer kontinuierlichen Sauerstoffzufuhr ist die Anlage eines transtrachealen $\mathrm{O}_{2}$-Katheters möglich. Als Sauerstoffquellen stehen zur Verfügung: Sauerstoffkonzentratoren, Sauerstoffdruckflaschen, Flüssigsauerstoffbehälter. $\mathrm{Zu}$ beachten sind bei der Sauerstofflangzeittherapie die Gefahr der $\mathrm{CO}_{2}$-Retention mit nachfolgender $\mathrm{CO}_{2}$-Narkose sowie die Austrocknung der Schleimhäute. Maßnahmen sind die sorgfältige Titrierung der Sauerstoffflussrate insbesondere bei COPD-Patienten sowie die Gabe von schleimhautschützenden Nasensalben oder Nasenölen.

In Analogie zu den COPD Patienten sollte auch bei anderen Formen pulmonaler Hypertonie bei Vorliegen einer arteriellen Hypoxämie eine $\mathrm{O}_{2}$-Langzeittherapie eingeleitet werden, auch wenn die Pathogenese der pulmonalen Hypertonie primär unabhängig von einer alveolären Hypoxie ist. Die Rationale hierfür besteht darin, den zusätzlichen Stimulus einer hypoxischen Vasokonstriktion bei schon bestehender pulmonaler Hypertonie und eine weitere Schädigung der ischämiebedrohten Organe (Herz, Nieren, Splanchnikusorgane) zu vermeiden. Randomisierte und kontrollierte Studien liegen hierzu jedoch nicht vor.

\section{Allgemeine Maßnahmen}

Neben der körperlichen Schonung gehören zu den allgemeinen Maßnahmen in Abhängigkeit von der Grunderkrankung und dem Stadium der Gefäßerkrankung verschiedene medikamentöse Maßnahmen, die einerseits auf die Rekompensation des Cor pulmonale, andererseits auf eine Hemmung thrombotischer oder embolischer Vorgänge in der Lungen- 
strombahn abzielen. Im Terminalstadium der Erkrankung kann eine Druckentlastung des rechten Ventrikels durch eine atriale Septostomie erfolgen, welche auch als Überbrückungsmaßnahme bis zur Lungentransplantation möglich ist. Diese Maßnahme ist allerdings bislang überwiegend bei jüngeren Patienten (unter 55 Jahre) mit schweren Formen der primären pulmonalen Hypertonie in spezialisierten Zentren zur Anwendung gekommen.

\section{Diuretika}

Unbestritten ist, dass ein dekompensiertes Cor pulmonale zur Volumenreduktion diuretisch behandelt werden muss. Eine Volumenretention im Endstadium der Erkrankung kann zu einer exzessiven Anhebung der rechtsventrikulären Vorlast führen mit der Konsequenz einer Leberstauung und den entsprechenden klinischen Befunden bis hin zum Leberversagen. Ödembildung und Aszites sind nicht nur auf die Rechtsherzinsuffizienz, sondern z.T. auch auf eine Stimulation des Renin-Angiotensin-Systems mit erhöhtem Aldosteronspiegel zurückzuführen. Somit kann eine Kombination der üblichen Diuretika mit Aldosteronantagonisten (z.B.: Spironolacton 50-200 mg/Tag) angezeigt sein. $\mathrm{Zu}$ beachten sind bei der diuretischen Therapie der chronischen pulmonalen Hypertonie die Risiken einer zu weitgehenden Entwässerung. Unterschreitet der rechtsventrikuläre Füllungsdruck eine kritische Schwelle, so kann der an die hohe Vorlast adaptierte rechte Ventrikel in seiner Leistung kritisch abnehmen, mit der Konsequenz eines Vorwärtsversagens. Eine engmaschige Steuerung der diuretischen Therapie ist somit um so notwendiger, je ausgeprägter die Rechtsherzinsuffizienz ist.

\section{Digitalis}

Strittig ist noch immer die Frage einer Digitalisierung bei Patienten mit chronischer pulmonaler Hypertonie. Nach einer randomisierten plazebokontrollierten Doppel-blind-Studie ist eine Digitalisierung beim chronischen Cor pulmonale nur dann erfolgversprechend, wenn gleichzeitig eine Einschränkung der linksventrikulären Funktion vorliegt. In jedem Fall ist eine Digitalisierung berechtigt, wenn eine tachykarde Form des Vorhofflimmerns behandlungsbedürftig erscheint. Es ist darauf zu achten, dass Digitalis gerade im Zusammenhang mit einer arteriellen Hypoxämie eine gesteigerte arrhythmogene Potenz besitzt, die zu erheblichen Komplikationen führen kann.

\section{Antikoagulation}

Nach Abschluss der üblichen Kontraindikation gilt die Antikoagulation mit Marcumar bzw. Heparin als gesichertes Therapieprinzip bei der schweren pulmonalen Hypertonie. Die Indikationsstellung basiert zum einen bei den thromboembolisch bedingten Unterformen der chronischen pulmonalen Hypertonie auf einer Reduktion des Rezidivrisikos, und bei den chronischen pulmonalen Hypertonieformen mit nicht thromboembolischer Genese auf den folgenden Aspekten:

- erhöhtes Risiko einer In-situ-Thrombosierung in der pulmonalen Strombahn durch den veränderten Blutfluss in den verengten und deformierten Lungengefäßen.

- erhöhtes Thromboserisiko im venösen System durch venöse Stase, Dilatation des rechten Ventrikels und Begrenzung der körperlichen Aktivität.
- Reduktion der zirkulierenden Thrombin- und Fibrinogenspaltprodukte, die als Wachstumsfaktoren an der Pathogenese des vaskulären Remodelling beteiligt sein könnten.

\section{Thrombendarteriektomie und Lungentransplantation}

Bei chronisch thromboembolischen Verschlüssen zentraler und/oder segmentaler bis subsegmentaler Lungengefäße als Ursache einer pulmonalen Hypertonie stellt die chirurgische Thrombendarteriektomie eine therapeutische Perspektive dar. Das perioperative Risiko dieser an nur wenigen spezialisierten Zentren erfolgreich durchgeführten Operation ist mit ca. $10 \%$ immer noch hoch, jedoch wird dadurch in den meisten Fällen eine weitgehende Verbesserung der Beschwerdesymptomatik bis hin zu weitgehender Symptomfreiheit erreicht. Sehr weit peripher lokalisierte thromboembolische Verschlüsse sind diesem Verfahren nicht zugänglich.

Bei Patienten mit primärer pulmonaler Hypertonie sind Einzel- und Doppellungentransplantationen und kombinierte Herz-Lungen-Transplantationen durchgeführt worden. Da die Überlebensrate nach internationalen Erfahrungen bei allen Verfahren ungefähr gleich ist, spricht die begrenzte Verfügbarkeit von Herz-Lungen-Transplantaten für eine bevorzugte Anwendung der Lungentransplantation. Selbst eine schon weitgehend eingeschränkte rechtsventrikuläre Pumpfunktion verbessert sich wesentlich sowohl nach einer einseitigen als auch nach einer beidseitigen Lungentransplantation. Die Einjahresüberlebensrate nach Lungentransplantationen wird für die primäre pulmonale Hypertonie zwischen 65 und $70 \%$ angegeben. Die Mortalitätsrate nach Lungentransplantationen scheint bei primärer pulmonaler Hypertonie höher zu liegen, als in anderen Patientenkollektiven. Somit muss die Entscheidung zur Lungentransplantation immer kritisch gegen die verbliebenen Möglichkeiten einer medikamentösen Therapie abgewogen werden. Bei Progredienz des Krankheitsbildes trotz maximaler Therapie stellt die Lungentransplantation jedoch die einzig verbleibende therapeutische Option dar. Die atriale Septostomie kann zur Entlastung des rechten Ventrikels und Verbesserung des Herzzeitvolumens Anwendung finden, jedoch um den Preis einer Verstärkung der arteriellen Hypoxämie auf Grund des Rechts-links-Shunts auf Vorhofebene. Sie ist nach Ausschöpfung aller medikamentösen Optionen im Vorfeld der Lungentransplantation indiziert [1].

\section{In der Entwicklung befindliche therapeutische Perspektiven bei der pulmonalen Hypertonie}

Ein neuer Ansatz mit dem Ziel, die Wirkdauer der Prostanoidtherapie zu verlängern, ist die Möglichkeit einer pharmakologischen Phosphodiesteraseinhibition mit pulmonaler Selektivität. Phosphodiesterasen (PDE) katalysieren den Abbau der second-messenger zyklisches Adenosinmonophosphat (cAMP) und Guanosinmonophosphat (cGMP) in den glatten Muskelzellen und limitieren daher den gefäßerweiternden Effekt der rezeptoroperierenden Vasodilatoren in verschiedenen Strombahnen. Eine Inhibition dieser Enzyme führt zu einem zytoplasmatischen Anstieg der cAMP- bzw. cGMP-Spiegel mit dem Resultat einer Vasodilatation [16]. Weiterhin wird die Sekretion von inflammatorischen Mediatoren in aktivierten Entzündungszellen gehemmt. Experimentell konnte an verschiedenen Modellen der pulmonalen Hypertonie durch eine spezifische Hemmung der Phosphodiesterase-Isoenzyme III, 

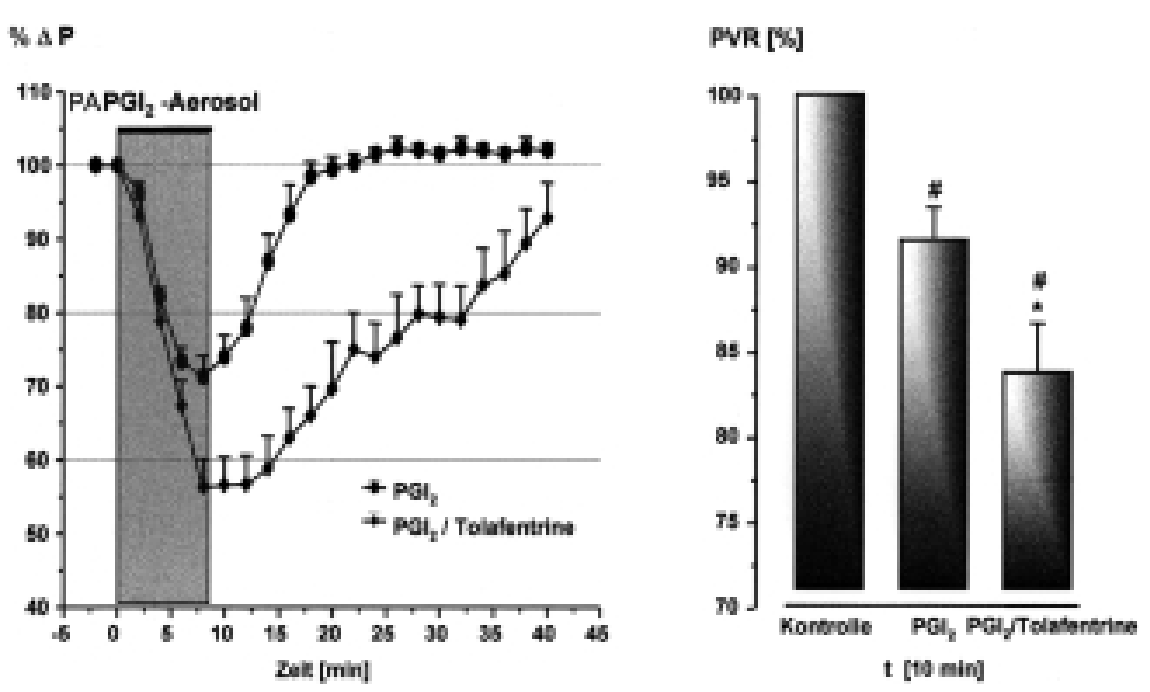

IV (cAMP) und V (cGMP) eine Amplifikation der prostanoidinduzierten pulmonalen Vasodilatation bereits gezeigt werden. Der Effekt für die Kombination von Prostazyklin mit dem PDE III/IV Inhibitor Tolafentrin ist in Abb. 7 dargestellt. Da für die klinische Anwendung bislang nur unspezifische mit erheblichen Nebenwirkungen belastete Phosphodiesterase-Inhibitoren verfügbar sind (Pentoxifyllin, Enoximon, Dipyridamol, Theophyllin), ist es das vorrangige Ziel aktueller Bemühungen, Phosphodiesterase-Inhibitoren mit optimaler pulmonaler Selektivität bei minimalen systemischen Nebenwirkungen zu entwickeln. Hierbei könnte wiederum der Aerosol-Applikation dieser Substanzen wesentliche Bedeutung zukommen.

\section{Literatur}

${ }^{1}$ The World Health Organization. World Symposium - Primary pulmonary hypertension. Executive Summary (Rich S, ed.). France: Evian, 1998

${ }^{2}$ Sitbon O, Humbert M, Jagot JL, Taravella O, Fartoukh M, Parent F, Herve P, Simonneau G. Inhaled nitric oxide as a screening agent for safely identifying responders to oral calcium-channel blockers in primary pulmonary hypertension. Eur Respir J 1998; 12(2): $265-270$

${ }^{3}$ Saadjian AY, Philip-Joet FF, Vestri R, Arnaud AG. Long-term treatment of chronic obstructive lung disease by nifedipine: an 18-month haemodynamic study. Europ Resp J 1998; 1: 716

${ }^{4}$ Rich S, Kaufmann E, Levy PS. The effect of high doses of calciumchannel blockers on surfvival in primary pulmonary hypertension. New Engl J Med 1992; 327: 76

${ }^{5}$ Erkan F, Cadvar T. Pulmonary vasculitis in Behcet's disease. Am Rev Respir Dis 1992; 146: 232 - 239

${ }^{6}$ Giaid A, Saleh D. Reduced expression of endothelial nitric oxide synthase in the lungs of patients with pulmonary hypertension. N Engl J Med 1995; 333: 214 - 221

${ }^{7}$ Brune B, Von-Knethen A, Sandau KB. Nitric oxide and its role in apoptosis. Eur J Pharmacol 1998; 351(3): 261 - 272

${ }^{8}$ Guo Y, DuVall MD, Crow JP, Matalon S. Nitric oxide inhibits $\mathrm{Na}+$ absorption across cultured alveolar type II monolayers. Am J Physiol 1998; 274(3 Pt 1): L369 - 377
Abb. 7 Kombination des Phosphodiesterase-Inhibitors Tolafentrin mit dem Prostazyklin. Dargestellt ist die Senkung des pulmonal-arteriellen Mitteldrucks (PAP) und des pulmonalvaskulären Widerstandes (PVR) in einem Model der in vivo induzierten pulmonalen Hypertonie des Kaninchens. Vor dem Experiment wurde der PAP durch eine Infusion mit dem Thromboxan-Agonisten U46619 auf ein konstantes Plateau erhöht (Anstieg des PAP von $\sim 13$ auf $\sim 30 \mathrm{mmHg}$ ). Die nachfolgende Kurzzeit-Aerosolierung mit Prostazyklin ( $56 \mathrm{ng} / \mathrm{kg} \mathrm{min}$ ) provozierte eine transiente Reduktion von PAP und PVR.

Durch den dualselektiven PDE III/IV Inhibitor Tolafentrin $\left(2 \mathrm{mg} / \mathrm{kg}^{*} \mathrm{~h}\right.$; diese Menge liegt unterhalb der Schwellendosis und ruft keinen eigenen pulmonalvasodilatativen Effekt hervor) konnte der Prostazyklin-Effekt amplifiziert und prolongiert werden. Dargestellt sind die Mittelwerte \pm SEM aus 8 unabhängigen Experimenten. \# signifikant gegenüber Kontrolle, ${ }^{*}$ signifikant gegenüber Prostazyklin.

${ }^{9}$ Haddad IY, Zhu S, Crow J, Barefield E, Gadilhe T, Matalon S. Inhibition of alveolar type II cell ATP and surfactant synthesis by nitric oxide. Am J Physiol 1996; 270(6 Pt 1): L898 - 906

${ }^{10}$ Higenbottam T, Cremona G, Voelkel NF, Black C, Rubin LJ, Olschewski $\mathrm{H}$, Lange PE. Advance in understanding and treatment of pulmonary hypertension. I. Epidemiology and pathophysiology. Eur Respir J, 1998. in press

${ }^{11}$ Barst RJ, Rubin LJ, Long Aand the pulmonary Hypertension Study Group. A comparison of continous intravenous epoprostenol (prostazyklin) with conventional therapy for primary pulmonary hypertension. N Engl J Med 1996; 334: 296 - 302

${ }^{12}$ Mc Laughlin VV, Rich S. Pulmonary hypertension-Advances in medical and surgical interventions. J Heart Lung Transplant 1998; 17: 739 - 743

${ }^{13}$ Rubin LJ. Primary pulmonary hypertension. N Engl J Med 1997; 336: $111-117$

${ }^{14}$ Olschewski H, Walmrath D, Schermuly R, Ghofrani A, Grimminger F, Seeger W. Aerosolized prostazyklin and iloprost in severe pulmonary hypertension. Ann Intern Med 1996; 124(9): $820-$ 824

15 Olschewski H, Ghofrani HA, Walmrath D, Schermuly R, Temmesfeld B, Grimminger F, Seeger W. Inhaled prostacyclin and iloprost in severe pulmonary hypertension secondary to lung fibrosis. Am J Respir Crit Care Med 1999; 160: 600 - 607

${ }^{16}$ Ichonese F, Adrie C, Hurford WE, Zapol WM. Prolonged pulmonary vasodilator action of inhaled nitric oxide by Zaprinast in awake lambs. J Appl. Physiol 1995; 78: 1288 - 1295

${ }^{17}$ Coleman RA, Smith WL, Narumiya S. International Union of Pharmacology classification of prostanoid receptors: properties, distribution, and structure of the receptors and their subtypes. Pharmacol Rev 1994; 46(2): 205 - 229

${ }^{18}$ Olschewski H, Walmrath D, Schermuly R, Ghofrani HA, Grimminger F, Seeger W. Aerosolised prostacyclin and iloprost in primary pulmonary hypertension. Ann Intern Med 1996; 124: $820-824$

Prof. Dr. Dr. F. Grimminger

Medizinische Klinik II

Klinikstraße 36

35392 Gießen 